International Journal of Algebra, Vol. 8, 2014, no. 18, 869 - 872

HIKARI Ltd, www.m-hikari.com

http://dx.doi.org/10.12988/ija.2014.410103

\title{
Monoids in Proximal Banach Spaces
}

\author{
J.F. Peters ${ }^{a, b, *}$, E. İnan ${ }^{a, b}$ and M.A. Öztürk ${ }^{a, b}$ \\ ${ }^{a}$ Computational Intelligence Laboratory \\ University of Manitoba, Winnipeg, MB R3T 5V6, Canada \\ * Corresponding author \\ ${ }^{b}$ Department of Mathematics, Faculty of Arts and Sciences \\ Adıyaman University, Adıyaman, Turkey
}

Copyright (c) 2014 J.F. Peters, E. İnan and M.A. Öztürk. This is an open access article distributed under the Creative Commons Attribution License, which permits unrestricted use, distribution, and reproduction in any medium, provided the original work is properly cited.

\begin{abstract}
Given the binary operation $\circ_{\delta}: 2^{V} \times 2^{V} \longrightarrow 2^{V}$ defined on a subset of $2^{V}$ in the proximal Banach space $V$, we prove that the monoid $S=2^{V}\left(\circ_{\delta}\right)$ is regular, every right ideal $A \subset S$ and left ideal $B \subset S$ are proximal, every ideal in $S$ is idempotent and $S$ is simple. For $A_{\circ}, B_{\circ}, C_{\circ} \in 2^{V}\left(\circ_{\delta}\right)$, we also prove that if $\left(\left(A_{\circ} \circ_{\delta} B_{\circ}\right) \circ_{\delta} C_{\circ}\right) \circ_{\delta} D_{\circ} \neq \emptyset$, then $D_{\circ}$ and at least one of the groupoids $A_{\circ}, B_{\circ}, C_{\circ}$ is proximal.
\end{abstract}

Mathematics Subject Classification: 20M32, 52A21, 54E05

Keywords: Banach space, Monoid, Proximity

\section{Introduction}

Monoids in proximal Banach spaces are introduced in this article, which is an outgrowth of recent work on proximity spaces $[5,9]$ and their applications $[7$, 8,6]. A monoid is a semigroup with an identity element. A Banach space is a complete, normed linear space. In this work, a Banach space is a Cech closure space $[10, \S 14$, p. 237$]$ endowed with a proximity relation. Results concerning the proximity of monoids in such spaces are given. 


\section{Preliminaries}

Let $V$ be a Banach space, $A, B \subset V, x, y \in V$. The Hausdorff distance $D(x, A)$ is defined by $D(x, A)=\inf \{\|x-y\|: y \in A\}$ and $\|x-y\|$ is the distance between vectors $x$ and $y$. The Cech closure [10] of $A$ (denoted by $\operatorname{cl} A$ ) is defined by $\operatorname{cl} A=\{x \in V: D(x, A)=0\}$. The sets $A$ and $B$ are proximal (near) (denoted $A \delta B$ ), provided $\operatorname{cl} A \cap \operatorname{cl} B \neq \emptyset[3]$. A Banach space endowed with the proximity relation $\delta$ is called a proximal Banach space (denoted by $(V, \delta))$.

Let $A \subset V$ be a nonempty subset of $V$. A binary operation $\circ: A \times A \rightarrow A$ maps $A \times A$ into $A$, where $A \times A$ is the set of all ordered pairs of elements of $A$ [2]. A groupoid in $V$ is a system defined by a nonempty set $A \subset V$ together with a binary operation $\circ$ on $A$ (denoted by $\left.A_{\circ}\right)$ such that $A_{\circ} \subseteq A$.

\section{Main Results}

Lemma 3.1. In a proximal Banach space $(V, \delta)$, let $A, B, C \subset V$ such that $B \subseteq(A \cup C)$. If $A \delta B$ and $B \delta C$, then $A \delta C$.

Proof. Case 1: Let $B \subseteq A, A \delta B$ and $B \delta C$. From $B \delta C, \operatorname{cl} B \cap \operatorname{cl} C \neq \emptyset$. Since $B \subseteq A, \operatorname{cl} B \subseteq \operatorname{cl} A$. Consequently, $\operatorname{cl} A \cap \operatorname{cl} C \neq \emptyset$. Hence, $A \delta C$. The other cases are similar.

From Lemma 3.1, obtain

Theorem 3.1. In a proximal Banach space $(V, \delta)$, let $A_{\circ}, B_{\circ}, C_{\circ} \subset V$ be groupoids such that $B_{\circ} \subseteq\left(A_{\circ} \cup C_{\circ}\right)$. If $A_{\circ} \delta B_{\circ}$ and $B_{\circ} \delta C_{\circ}$, then $A_{\circ} \delta C_{\circ}$.

Let $2^{V}$ be the set of all subsets of $V, A, B \in 2^{V}$, and let

$$
\delta=\left\{(A, B) \in 2^{V} \times 2^{V}: \operatorname{cl} A \cap \operatorname{cl} B \neq \emptyset\right\} .
$$

Consider the binary operation $\circ_{\delta}: 2^{V} \times 2^{V} \longrightarrow 2^{V}$ defined on a subset of $2^{V}$, where

$$
A \circ_{\delta} B= \begin{cases}A \cap B, & \text { if } A \delta B, \\ \emptyset, & \text { otherwise. }\end{cases}
$$

It is easily shown that $\left(2^{V}, o_{\delta}\right)$ is a commutative monoid such that $V \in 2^{V}$ is the identity element of the monoid. And $\left(2^{V}, o_{\delta}\right)$ is called a proximal monoid in $V$ (briefly, $2^{V}\left(o_{\delta}\right)$ ).

The following Theorem from K. Iséki [4] (see also [1]) serves as a characterization of regular monoids in proximal Banach spaces.

Theorem 3.2. (Iséki [4]) Any semigroup $S(\cdot)$ is regular if and only if $A \cdot B=$ $A \cap B$ for every right ideal $A \subset S$ and left ideal $B \subset S$. 
Theorem 3.3. Let $S=2^{V}\left(\circ_{\delta}\right)$ be a monoid in a proximal Banach space $(V, \delta)$. The following statements are equivalent.

$1^{\circ} S$ is regular.

$2^{\circ} A \circ_{\delta} B=A \cap B$ for every right ideal $A \subset S$ and left ideal $B \subset S$.

$3^{\circ}$ Every ideal in $S$ is idempotent [4, Theorem 2, p. 676].

$4^{\circ} S$ is simple, i.e., $S$ itself is the only ideal of $S$ [2, p. 4].

Proof. We only prove $2 \Leftrightarrow 1$ : Let $B, A \subset S$ be left and right ideals, respectively.

$$
A \circ_{\delta} S \circ_{\delta} B=A \circ_{\delta} B \subseteq B \Rightarrow A \delta B \Rightarrow A \circ_{\delta} B=A \cap B \Leftrightarrow
$$

from Theorem $3.2, S$ is regular.

Lemma 3.2. Let $(V, \delta)$ be a proximal Banach space, $A, B, C \in 2^{V}\left(\circ_{\delta}\right)$. If $\left(A \circ_{\delta} B\right) \circ_{\delta} C \neq \emptyset$, then $A \delta C$ or $B \delta C$.

Proof. Let $\left(A \circ_{\delta} B\right) \circ_{\delta} C \neq \emptyset$. Then $\left(A \circ_{\delta} B\right) \circ_{\delta} C=(A \cap B) \cap C$. From the definition of $\circ_{\delta},(A \cap B) \delta C$, i.e., $\operatorname{cl}(A \cap B) \cap \operatorname{cl} C \neq \emptyset$. Since $\operatorname{cl}(A \cap B) \subseteq \operatorname{cl} A$ or $\operatorname{cl}(A \cap B) \subseteq \operatorname{cl} B$, then $\operatorname{cl} A \cap \operatorname{cl} C \neq \emptyset$ or $\operatorname{cl} B \cap \operatorname{cl} C \neq \emptyset$. Hence $A \delta C$ or $B \delta C$.

Theorem 3.4. Let $(V, \delta)$ be a proximal Banach space, $A_{\circ}, B_{\circ}, C_{\circ} \in 2^{V}\left(\circ_{\delta}\right)$. If $\left(A_{\circ} \circ_{\delta} B_{\circ}\right) \circ_{\delta} C_{\circ} \neq \emptyset$, then $A_{\circ} \delta C_{\circ}$ or $B_{\circ} \delta C_{\circ}$.

Proof. Immediate from Lemma 3.2.

Lemma 3.3. Let $(V, \delta)$ be a proximal Banach space, $A, B, C \in 2^{V}\left(\circ_{\delta}\right)$. If $\left(\left(A \circ_{\delta} B\right) \circ_{\delta} C\right) \circ_{\delta} D \neq \emptyset$, then $D$ and at least one of the sets $A, B, C$ are proximal.

Proof. Immediate from Lemma 3.2.

From Lemma 3.3, obtain

Theorem 3.5. Let $(V, \delta)$ be a proximal Banach space and $A_{\circ}, B_{\circ}, C_{\circ} \in 2^{V}\left(\circ_{\delta}\right)$. If $\left(\left(A_{\circ} \circ_{\delta} B_{\circ}\right) \circ_{\delta} C_{\circ}\right) \circ_{\delta} D_{\circ} \neq \emptyset$, then $D_{\circ}$ and at least one of the groupoids $A_{\circ}, B_{\circ}, C_{\circ}$ is proximal.

Acknowledgements. This research has been supported by the The Scientific and Technological Research Council of Turkey (TÜBITAK) Scientific Human Resources Development (BIDEB) under grants: 2221-1059B211301223, 2221-1059B211402463 and Natural Sciences \& Engineering Research Council of Canada (NSERC) discovery grant 185986. 


\section{References}

[1] J. Chvalina, The Iséki characterization of certain regular ordered semigroups, Czech. Math. Journal, 47 (1997), no. 122, 261-276, Zbl 0934.20051.

[2] A.H. Clifford and G.B. Preston, The algebraic theory of semigroups I, American Mathematical Society, Providence, RI, 1964, MR0132791.

[3] A. Di Concilio, Proximity: A powerful tool in extension theory, function spaces, hyper-spaces, boolean algebras and point-free geometry, Contemporary Math. 486 (2009), 89114, MR2521943.

http://dx.doi.org/10.1090/conm/486/09508

[4] K. Iséki, A characterization of regular semi-groups, Proc. Japan Academy, 32 (1956), no. 9, 676-677, Zbl 0072.25604. http://dx.doi.org/10.3792/pja/1195525246

[5] S.A. Naimpally and J.F. Peters, Preservation of continuity, Scientiae Math. Japonicae, 76 (2013), no. 2, 305-311.

[6] J.F. Peters, Topology of Digital Images. Visual Pattern Discovery in Proximity Spaces, Springer, Berlin, 2014, xv + 411pp, Zentralblatt MATH Zbl 1295.68010 .

[7] J.F. Peters, Local near sets: Pattern discovery in proximity spaces, Math. in Comp. Sci., 7 (2013), no. 1, 87106, MR3113385.

http://dx.doi.org/10.1007/s11786-013-0143-z

[8] J.F. Peters and S.A. Naimpally, Applications of near sets, Notices of the Amer. Math. Soc. 59 (2012), no. 4, 536-542, MR2951956.

[9] J.F. Peters and E. İnan and M.A. Öztürk, Spatial and Descriptive Isometries in Proximity Spaces, Gen. Math. Notes, 21 (2014), no. 2, 125-134.

[10] E. C̆ech, Topological Spaces, revised Ed. by Z. Frolik and M. Katĕtov, John Wiley \& Sons, London, 1966, MR0104205.

Received: October 23, 2014; Published: December 3, 2014 